\title{
Original
}

\section{Rewarming Injury against the Graft Liver in Orthotopic Liver Transplantation}

\author{
Masahiro HAYASHI, Yoshinori SHIMIZU, \\ Masahiko Murakami and Mitsuo Kusano
}

\begin{abstract}
We investigated the effect of rewarming injury on the graft liver in rats. We extended the anhepatic phase to 40 minutes and the fixed rewarming time at the usual 15 minutes (group $\mathrm{A}, \mathrm{n}=7$ ) or at 35 minutes (group $\mathrm{B}$, $\mathrm{n}=6$ ). Graft temperatures were measured at various times after transplantation. Survival, serum levels of aspartate aminotransferase, alanine aminotransferase, alkaline phosphatase, and hyaluronic acid, and histologic findings were compared between groups A and group B. The graft temperature 15 minutes after transplantation $\left(22.7 \pm 0.7^{\circ} \mathrm{C}\right)$ was significantly lower than that after 35 minutes $\left(27.7 \pm 0.8^{\circ} \mathrm{C}\right)$. Survival was significantly lower in group $\mathrm{A}$ and serum levels of aspartate aminotransferase and alanine aminotransferase were significantly higher in group B. Histologic findings in group A were nearly normal, but severe damage to hepatocytes and sinusoids was observed in group B. We conclude that rewarming injures hepatocytes and sinusoids; therefore, rewarming injury affects graft viability. Furthermore, we speculate that cooling below $23^{\circ} \mathrm{C}$ during transplantation might be necessary for maintaining graft viability.
\end{abstract}

Key words : rat liver transplantation, rewarming time, anhepatic phase, graft temperature, graft viability

\section{Introduction}

Since the cuff technique was established, the rat model of orthotopic liver transplantation (OLT) has been used to investigate ischemic, preservative, and immunologic aspects of liver transplantation $^{1,2,4)}$. In the rat model, injuries caused by ischemia, preservative temperature, and the method of harvest affect graft survival ${ }^{5)}$. Moreover, we and others have recently reported that the rewarmed graft liver is injured and that rewarming injury affects rat survival in $\mathrm{OLT}^{6,7)}$. In this study, we investigated rewarming injury, a type of warm ischemic injury, and investigated the effect of rewarming injury on the graft liver.

\section{Materials and Methods}

We used male Wistar rats weighing about $250 \mathrm{~g}$ as both donors and recipients. The graft livers were preserved in lactated Ringers solution at $4{ }^{\circ} \mathrm{C}$ for about 1 hour before OLT. In rat liver transplantation, the anhepatic phase $(\mathrm{AH})$ is usually 20 minutes and rewarming 


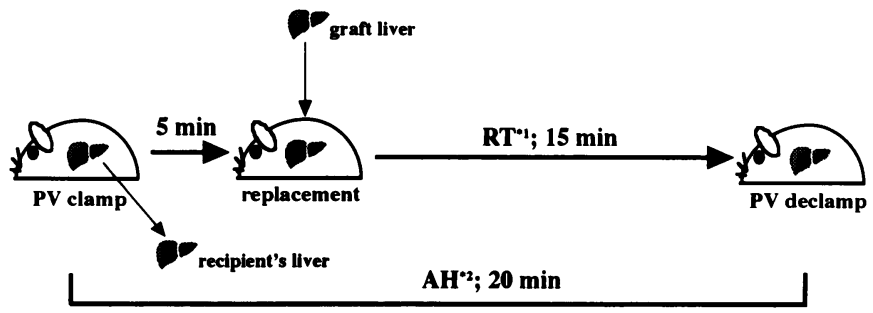

*1 RT; rewarming time

* 2 AH; anhepatic phase

Fig. 1. Usual orthotopic rat liver transplantation. In usual rat liver transplantation, $\mathrm{RT}$ and $\mathrm{AH}$ are 15 minutes and 20 minutes, respectively.
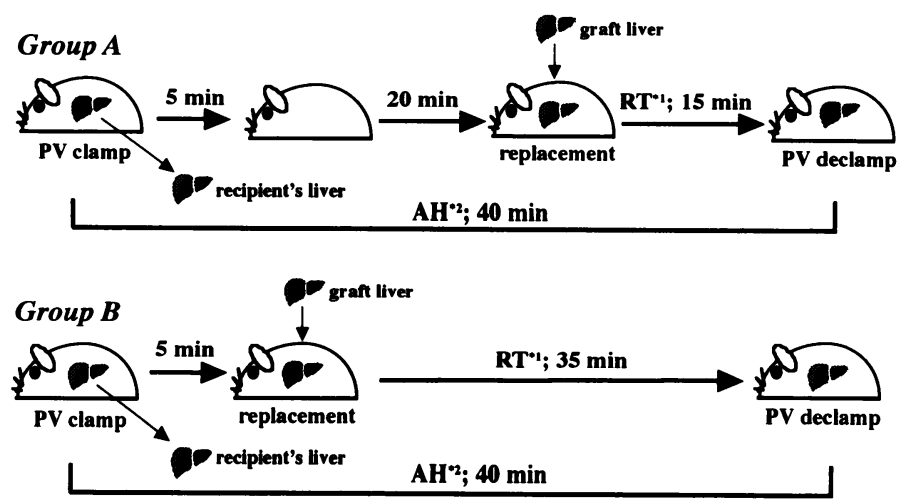

*1 RT; rewarming time

*2 AH; anhepatic phase

Fig. 2. Timing of graft liver transplantation.

AH was fixed at 40 minutes. In group A, RT was the usual 15 minutes and in group B, RT was extended to 35 minutes.

time $(\mathrm{RT})$ is 15 minutes, since the recipients liver is first removed for 5 minutes, after which, the graft liver is transplanted and the suprahepatic inferior vena cava and portal vein are reconstructed for 15 minutes (Fig. 1). In this study, we prolonged AH to 40 minutes and fixed the RT at the usual 15 minutes (group $A, n=7$ ) or 35 minutes (group $B, n=6$ ) to evaluate the effect of rewarming injury (Fig. 2).

To measure graft temperatures, we used a wire probe thermometer (Model TM-54H, Inter Nova Corp., Tokyo). The probe was inserted into the center of the right lobe of the graft liver to a depth of $5 \mathrm{~mm}$.

One milliliter of blood was collected 1 day after OLT, and serum levels of aspartate aminotransferase (AST), alanine aminotransferase (ALT), alkaline phosphatase (ALP), and hyaluronic acid (HA) were analyzed. Implanted livers were removed 1 day after OLT for histologic examination with hematoxylin-eosin stain. The serum levels of AST, ALT, ALP and $H A$ are presented as means $\pm S E$ and differences between groups were analyzed with the Mann-Whitney $U$ test. Statistical analysis ofdifferences in graft temperature at 15 and 35 minutes, and survival between groups $\mathbf{A}$ and $\mathbf{B}$ were performed with the paired t-test and 


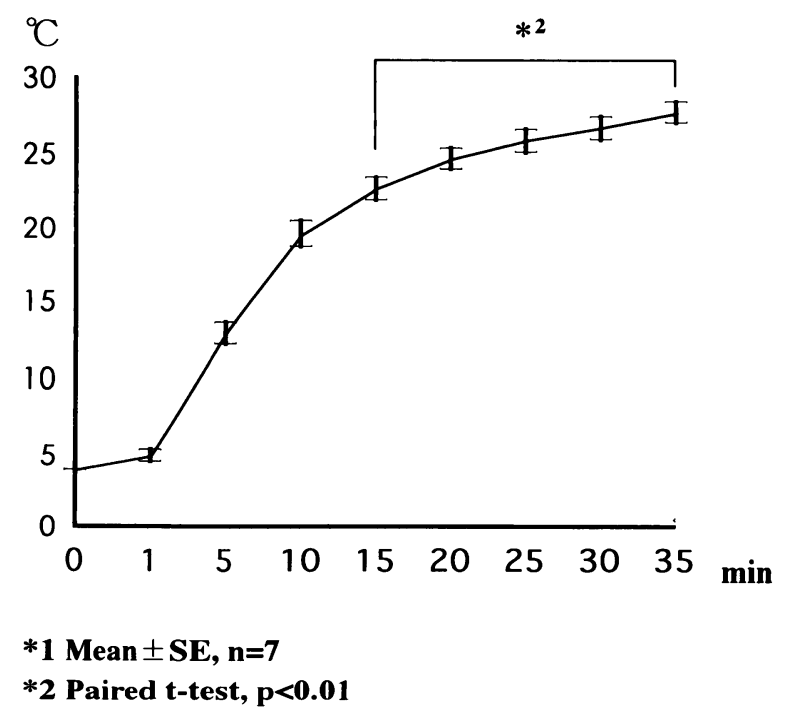

Fig. 3. Graft temperatures were measured in seven cases after transplantation $($ mean $\pm \mathrm{SE})$. Graft temperatures at 15 minutes and at 35 minutes differed (paired t-test, $\mathrm{p}<0.01$ ).

Table. Serum levers of AST, ALT, ALP, and HA

\begin{tabular}{lrcc}
\hline & Group A & Group B & p value * \\
\hline AST $(\mathrm{U} / \mathrm{ml})$ & $497.0 \pm 123.1$ & $2681.0 \pm 179.2$ & 0.0027 \\
ALT $(\mathrm{U} / \mathrm{l})$ & $1330.0 \pm 207.8$ & $7640.6 \pm 1312.5$ & 0.0027 \\
ALP $(\mathrm{IU} / \mathrm{l})$ & $585.5 \pm 96.8$ & $948.7 \pm 159.3$ & 0.09 \\
HA $(\mathrm{ng} / \mathrm{ml})$ & $298.0 \pm 40.7$ & $644.3 \pm 217.4$ & 0.15 \\
\hline
\end{tabular}

*Mann-Whitney U test

Fishers test, respectively.

\section{Results}

1. Graft temperature

The graft temperature increased from $4^{\circ} \mathrm{C}$ to $22.7 \pm 0.7^{\circ} \mathrm{C}$ at 15 minutes and finally to $27.7 \pm 0.8^{\circ} \mathrm{C}$ at 35 minutes after transplantation. Graft temperatures at 15 minutes and at 35 minutes differed significantly (paired t-test) (Fig. 3).

2. Survival

In group A, all rats survived more than 60 days after OLT, but in group B, all rats died within 2 days. Survival in groups A and B differed significantly (Fishers test).

3. Serum levels of AST, ALT, ALP, and HA

Serum levels of AST and ALT were significantly higher in group B than in group A (Table 1). However, serum levels of ALP and HA did not differ significantly between the two groups.

4. Histologic findings

No marked changes in hepatocytes and sinusoids were found in group A (Fig. 4a), but 


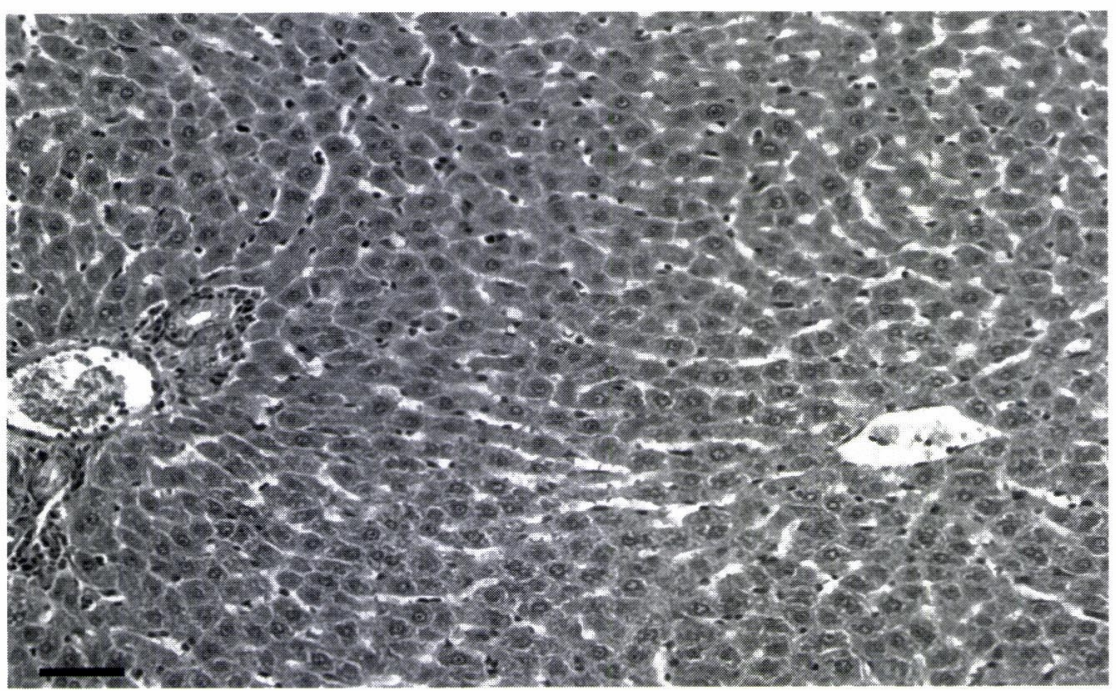

Fig. 4a Microscopic findings in Group A

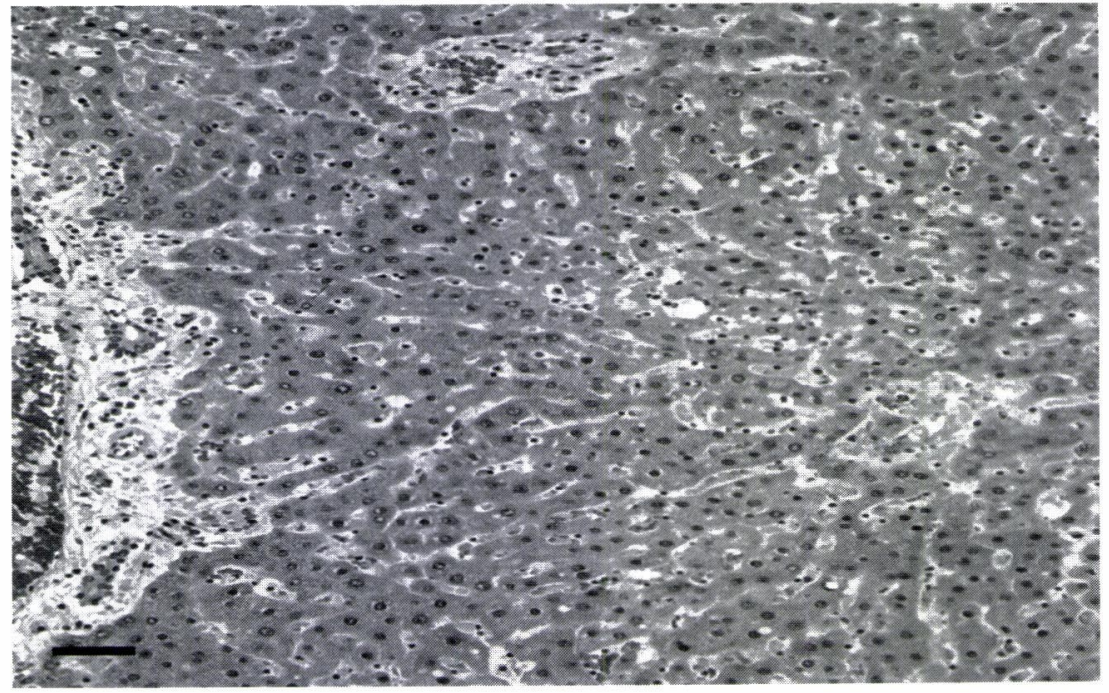

Fig. 4b Microscopic findings in Group B

Fig. 4. Sections of graft liver stained with hematoxylin-eosin

1 day after OLT were almost normal in Group A (Fig. 4a, $\times 200$ ). Bars, $100 \mu \mathrm{m}$. However, there were severe degenerative changes, ballooning and necrosis in hepatocytes, and dilatation of sinusoids, in Group B (Fig. 4b, $\times 200$ ). Bars, $100 \mu \mathrm{m}$.

severe degenerative changes,such as ballooning and necrosis of hepatocytes and dilatation of sinusoids, were observed in group B (Fig. 4b).

\section{Discussion}

Although OLT is the definitive treatment for hepatic diseases when medical or surgical treatment has not been effective ${ }^{8)}$, technical difficulties ${ }^{9)}$, rejection ${ }^{10)}$, and ischemic 
reperfusion injuries may prevent its use ${ }^{11)}$. Therefore, to investigate ischemic, preservative, and immunologic aspects of OLT, rat models of OLT have been established ${ }^{1-4)}$.

Recently, some types of injury to the graft liver have been studied using rat models ${ }^{12}$. We have also reported that rewarming injury, a type of ischemic injury, effects survival in rat liver transplantation ${ }^{7)}$. Clavien et al. first reported rewarming injury ${ }^{6}$, but mechanisms of rewarming injury are still unknown. In this study, we studied the effects of temperature and rewarming condition on graft liver injury.

We speculate two mechanisms of injury from rewarming times: one is a rapid increase in temperature and another is limits of temperature. In this study, group A $(R T=15$ minutes) was not severely injured regardless whether the graft temperature was suddenly increased from 1 minute to 15 minutes. These results suggest the existence of graft temperature limits.

The graft temperature gradually incresed from $4{ }^{\circ} \mathrm{C}$ to about $23^{\circ} \mathrm{C}$ at 15 minutes after transplantation, and all recipients in which $\mathrm{RT}$ was 15 minutes survived more than 60 days.

In contrast, the graft temperature was about $28^{\circ} \mathrm{C}$ at 35 minutes after transplantation and all rats in which RT was 35 minutes died within 2 days after OLT. Moreover, when RT was 15 minutes (group A), the serum levels of AST and ALT were higher than those in group B (RT, 35 minutes). As a result, when the graft temperature was $23^{\circ} \mathrm{C}$, graft viability was maintained, suggesting that the graft liver should be cooled to less than 23 ${ }^{\circ} \mathrm{C}$ to maintain graft viability.

Injury to hepatocytes and sinusoids was slight when RT was 15 minutes, but was severe when RT was 35 minutes. However, serum levels of HA, a marker of sinusoidal viability, did not differ significantly between group $A(R T=15$ minutes) and group $B \quad(R T=35$ minutes). This result suggests that rewarming injury effects both hepatocytes and sinusoids, although ischemic injury mainly affects sinusoids ${ }^{12}$.

\section{Conclusion}

1) The graft temperatures were $22.7 \pm 0.7^{\circ} \mathrm{C}$ at 15 minutes and $27.7 \pm 0.8^{\circ} \mathrm{C}$ at 35 minutes after transplantation.

2) When RT was 35 minutes, the serum levels of ALT and AST were significantly higher than those when RT was 15 minutes.

3) When RT was 35 minutes, severe damage of both hepatocytes and sinusoids was seen, although hepatocytes and sinusoids were almost completely normal when RT was 15 minutes.

\section{References}

1) Kamada $\mathbf{N}$ and Calne RY: Orthotopic liver transplantation in the rat. Technique using cuff for portal vein anastomosis and biliary drainage. Transplantation, $28: 47-50$ (1979)

2) Kamada N and Calne RY: A surgical experience with 530 liver transplantation in the rat. Surgery, 79:64-69 (1983)

3) Lee S, Charter AC, Chandler JG and Orloff NJ:A technique for orthotopic liver transplantation in the rat. Transplantation, 16 : 664-669 (1973)

4) Kobayashi E, Kamada N, Goto S and Miyata M : Protocol for the technique of orthotopic liver transplantation in the rat. Microsurgery, $14: 1-6$ (1993)

5) Goto M, Takei Y, Kawano S, Yoshiyama H, Fukai H, Matsunaga T, Oshita M, Nishimura Y, Fusamoto H and Kamada T: Endothelin-1 antibody improved hepatic microcirculatory disturbance and damage after ischemia reflow procedure. Hepatology, $14: 59 \mathrm{~A}$ (1991)

6) Clavien PA, Harvey PRC and Strasberg AM: Preservation and reperfusion injuries in liver allografts. 
Transplantation, 53 : 957-978 (1992)

7) Shimizu Y, Goto S, Chiba S, Kamada N, Murakami M and Kusano M: Orthotopic liver transplantation in rats with special reference to anhepatic phase and rewarming time. Jpn.J. Transplant, 31:89-93 (1996) (in Japanese)

8) Iwatsuki S, Starzl TE, Sheahan DG, Yokoyama I, Demetris AJ, Todo S, Tzakis AG, Van Thiel DH, Carr B and Selby R: Hepatic resection versus transplantation for hepatocellular carcinoma. Ann Surg, 214: 221-228 (1991)

9) Starzl TE, Porter KA, Putnam CW, Schroter GP, Halgrimson CG, Weil R 3d, Hoelscher M and Reid HA : Orthotopic liver transplantation in 93 patients. Surg Gynecol Obstet, 142 : 487-505 (1976)

10) Seiichi S :Transplantation immunnology and immunosurpressive drug. J Jpn Surg Soc, 11 : 964-969 (1996) (in Japanese)

11) den Butter G, Marsh DC, Lindell SL, Southard JH and Belzer FO : Amino acids to suppress reperfusion injury after liver prreservation. Transplant Proc, $23: 2378$ (1991)

12) Morimoto $T$, Kusumoto $\mathrm{K}$ and Isselhard $\mathrm{W}$ : Impairment of grafts by short-term warm ischemia in rat liver transplantation. Transplantation, $52:$ 424-431 (1991)

[Received March 4, 1998 : Accepted March 6, 1998] 